\title{
Heat capacity and X-ray scattering studies on phase transitions of $\mathrm{Rb}_{2} \mathrm{MoO}_{4}$
}

\author{
Hirotake Shigematsua, Shinya Kaneyasua, Atsuko Uchidab, Hitoshi Kawaji ${ }^{b}$, \\ Hironobu Kasanoc, and Hiroyuki Mashiyamad \\ aFaculty of Education, Yamaguchi University, Yamaguchi, Japan; \\ bMaterials and Structures Laboratory, Tokyo Institute of Technology, Yokohama, Japan; \\ cDepartment of Physics, Faculty of Science, Yamaguchi University, Yamaguchi, Japan; \\ dYamaguchi University, Yamaguchi, Japan
}

(Received 19 June 2016, Accepted 27 March 2017, Published online: 07 Sep 2017.)

\begin{abstract}
The structural phase transitions of $\mathrm{Rb}_{2} \mathrm{MoO}_{4}$ have been reinvestigated using heat capacity measurement and X-ray scattering technique. In as-grown single crystals of $\mathrm{Rb}_{2} \mathrm{MoO}_{4}$, the crystal structure is a monoclinic one isomorphous to $\beta-\mathrm{K}_{2} \mathrm{MoO}_{4}$ ( $\alpha$-sequence) at room temperature. The $\alpha$-sequence transforms into the $\beta$-sequence (isomorohius to $\beta-\mathrm{K}_{2} \mathrm{SO}_{4}$, space group Pnam) at a temperature range from 400 to $500 \mathrm{~K}$. This $\alpha-\beta$ transition is reconstructive and depends on both temperature and time, especially with accompanying the temperaturedependent incubation time. In the $\beta$-sequence, an incommensurate phase characterized by wave vector $q_{\mathrm{x}}=1 / 3 \sim 2 / 5$ was observed below $T_{3}=223 \mathrm{~K}$.
\end{abstract}

\section{Introduction}

A lot of $\mathrm{A}_{2} \mathrm{BO}_{4}$-type dielectrics, for example, $\mathrm{A}_{2} \mathrm{SO}_{4}, \mathrm{~A}_{2} \mathrm{CrO}_{4}, \mathrm{~A}_{2} \mathrm{MnO}_{4}, \mathrm{Rb}_{2} \mathrm{SeO}_{4}, \mathrm{Cs}{ }_{2} \mathrm{SeO}_{4}$, $\mathrm{Cs}_{2} \mathrm{MoO}_{4}$, and $\mathrm{Cs}_{2} \mathrm{WO}_{4}$ (here $\mathrm{A}=\mathrm{K}, \mathrm{Rb}$, and $\mathrm{Cs}$ ), undergo a same type of successive phase transition (group I): these transform from the parent high-symmetry phase (phase I, space group $P 6_{3 / m m c} \alpha-\mathrm{K}_{2} \mathrm{SO}_{4}$ type structure) to the orthorhombic phase (phase II, Pnam, $\beta-\mathrm{K}_{2} \mathrm{SO}_{4}$ type structure) at high temperature $(T$-II $=700 \sim 1100 \mathrm{~K})$, and retain the orthorhombic system down to $0 \mathrm{~K}$. In $\mathrm{K}_{2} \mathrm{SeO}_{4}$, with decreasing temperature, the crystal transforms from phase I to phase II, and to an incommensurate phase (phase III), which is followed by a ferroelectric phase (Phase IV, Pna2 ${ }_{1}$ ) [1]. Among these $\mathrm{A}_{2} \mathrm{BO}_{4}$-type dielectrics belonging to the group I, it has been believed that only $\mathrm{K}_{2} \mathrm{SeO}_{4}$ transforms into the incommensurate phase. It is suggested that the other oxides in group I have no transition below the phase II; that is, if the phase II structure became unstable, then it took place below $0 \mathrm{~K}$ [2-4].

On the other hand, another group in $\mathrm{A}_{2} \mathrm{BO}_{4}$-type dielectrics (group II), e.g. $\mathrm{K}_{2} \mathrm{MoO}_{4}, \mathrm{~K}_{2} \mathrm{WO}_{4}$, $\mathrm{Rb}_{2} \mathrm{MoO}_{4}$, and $\mathrm{Rb}_{2} \mathrm{WO}_{4}$, take the monoclinic $\beta-\mathrm{K}_{2} \mathrm{MoO}_{4}$ type structure (space group $C 2 / m$ ) at room temperature and two typical first-order anomalies were observed above room temperature by differential scanning calorimetry (DSC) measurement [5]. Furthermore, in $\mathrm{Rb}_{2} \mathrm{MoO}_{4}$, the existence of polymorphous structures has been confirmed at room temperature 
[6]. One of the polymorphous structures is the monoclinic $\beta-\mathrm{K}_{2} \mathrm{MoO}_{4}$ type one called as the $\alpha^{-}$ sequence and another is the orthorhombic $\beta-\mathrm{K}_{2} \mathrm{SO}_{4}$ type one called as the $\beta$-sequence. In the $\beta$ sequence, a new $\lambda$-type anomaly similar to the normal-incommensurate (N-INC) phase transition in $\mathrm{K}_{2} \mathrm{SeO}_{4}$ was observed at $\mathrm{T}_{3}=223 \mathrm{~K}$. According to an empirical rule about the relation between the lattice parameter ratios $a_{0} / c_{0}$ and the N-INC phase transition temperatures $[6,7]$, we can expect that the incommensurate phase should exist in the $\beta^{-}$ sequence of $\mathrm{Rb}_{2} \mathrm{MoO}_{4}$. However, behavior of modulation in the incommensurate phase, the presence or absence of the lock-in transition, and mechanism of the transition between $\alpha^{-}$and $\beta$-sequences ( $\alpha-\beta$ transition) have not yet been clarified definitely in $\mathrm{Rb}_{2} \mathrm{MoO}_{4}$ so far. In order to obtain additional information about structures and structural phase transitions in $\mathrm{Rb}_{2} \mathrm{MoO}_{4}$, we have performed heat capacity measurement and X-ray scattering experiments.

\section{Experimental}

Single crystals of $\mathrm{Rb}_{2} \mathrm{MoO}_{4}$ were grown by a slow evaporation method from a saturated ammonium solution of $\mathrm{Rb}_{2} \mathrm{CO}_{3}$ and $\mathrm{MoO}_{3}$ at $310 \mathrm{~K}$. As-grown samples have the monoclinic $\mathrm{b}^{-}$ $\mathrm{K}_{2} \mathrm{MoO}_{4}$ type structures (space group $\mathrm{C} 2 / \mathrm{m}$ ) at room temperature. The existence of polymorphous structures has been confirmed. It is also confirmed that annealed samples, kept in air at $500 \mathrm{~K}$ for 4 hours, have the orthorhombic morphotype ( $\beta-\mathrm{K}_{2} \mathrm{SO}_{4}$ type structure, space group Pnam). All obtained samples were colorless and transparent and showed strong deliquescence.

A heat capacity measurement was carried out using a heat capacity measurement module, Quantum Design PPMS, in the temperature range from 2 to $300 \mathrm{~K}$; the sample weights were $4.02 \mathrm{mg}$ for as-grown $\mathrm{Rb}_{2} \mathrm{MoO} 4$. Above $300 \mathrm{~K}$, differential scanning calorimetry (DSC) measurement was carried out on a SEIKO DSC220 with a heating rate of $5 \mathrm{Kmin}^{-1}$. X-ray powder diffraction measurements were performed using RIGAKU Smart Lab XRD spectrometer with $\mathrm{Cu}-\mathrm{K} \alpha$ radiation. Furthermore, the diffraction data for single crystals were collected using an automatic four-circle diffractometer (RIGAKU AFC-5R) with graphitemonochromated Mo-K $\alpha$ radiation. The temperature of the specimen was controlled within +$0.1 \mathrm{~K}$ using a cold nitrogen gas flow system.

\section{Results and discussion}

Figure 1 shows the molar heat capacity measured below room temperature in as-grown $\mathrm{Rb}_{2} \mathrm{MoO}_{4}$ ( $\alpha$-sequence) and annealed $\mathrm{Rb}_{2} \mathrm{MoO}_{4}$ ( $\beta$-sequence). In as-grown $\mathrm{Rb}_{2} \mathrm{MoO}_{4}$, no anomalous change is detectable below room temperature (Fig. 1(a)). Furthermore, the X-ray diffraction pattern corresponds to the $\beta-\mathrm{K}_{2} \mathrm{MoO}_{4}$ type structure (space group $C 2 / \mathrm{m}$ ) at room temperature and the pattern remains unchanged below room temperature (phase $\mathrm{I}_{\alpha}$ ).

On the other hand, in annealed $\mathrm{Rb}_{2} \mathrm{MoO}_{4}$, the $\lambda$-type anomaly in the molar heat capacity is observable at $\mathrm{T}_{3}=223 \mathrm{~K}$ [7]. The X-ray diffraction pattern that corresponds to the $\beta-\mathrm{K}_{2} \mathrm{SO}_{4}$ type structure (space group Pnam) at room temperature (phase $\mathrm{III}_{\beta}$ ).

Below $T_{3}$ (phase $\mathrm{IV}_{\beta}$ ), a new incommensurate phase characterized by wave vector $q_{\mathrm{x}}=1 / 3 \sim 2 / 5$ 
was observed by single-crystal X-ray diffraction technique. Figure 2 shows the integrated intensity $I$ and the satellite position of the $h 32$ superlattice reflection, where indices are referred to the normal phase $\mathrm{III}_{\beta}$, on cooling. The temperature dependence of I can be fitted to the relation $I=A\left(T_{3}-T\right)^{2 \beta}$ where $A$ denotes the constant and the critical index $\beta$ is estimated as 0.36. The modulation wave vector $q_{x}$ is close to $1 / 3$ at $T_{3}$ and changes monotonously approaching to $2 / 5$, with decreasing temperature. The presence or absence of the lock-in transition was not able to confirm, because of the limit of the measurement temperature under a condition using a cold nitrogen gas flow system.

In the case of $\mathrm{K}_{2} \mathrm{SeO}_{4}$, the modulation wave number $q_{x}$ changes from 0.31 to 0.33 in the incommensurate phase and it locks into the commensurate value $1 / 3$; the phase is ferroelectric (space group Pna21). However, the behavior of the modulation wave number is different between $\mathrm{K}_{2} \mathrm{SeO}_{4}$ and $\mathrm{Rb}_{2} \mathrm{MoO}_{4}$; the wave number $q_{x}$ approaches to $1 / 3$ in the former, but to $2 / 5$ in the latter. The crystal structure of the commensurate phase in $\mathrm{Rb}_{2} \mathrm{MoO}_{4}$ will probably be different from that of $\mathrm{K}_{2} \mathrm{SeO}_{4}$ if the lock-in transition exists in $\mathrm{Rb}_{2} \mathrm{MoO}_{4}$ [8].

Another interesting characteristic of $\mathrm{Rb}_{2} \mathrm{MoO}_{4}$ is that the $\alpha-\beta$ reconstructive transition takes place between polymorphous structures, which is described as follows. Figure 3(a) shows the temperature dependence of the integrated intensities of the Bragg reflections 312 in phase $I_{\alpha}$ of $\alpha$-sequence and 111 in phase $\mathrm{III}_{\beta}$ of $\beta$-sequence for the as-grown $\mathrm{Rb}_{2} \mathrm{MoO}_{4}$ crystal with the average heating rate of $10 \mathrm{~K} / \mathrm{min}$. The intensities of the Bragg reflections of two polymorphous structures change gradually and coexist in a temperature range of about $100 \mathrm{~K}$ similarly.

The as-grown crystal was heated from room temperature with a speed of $10 \mathrm{~K} / \mathrm{min}$ and the temperature was kept at $450 \mathrm{~K}$. Then the integrated intensities of Bragg reflections changed with time. The time dependence is shown Fig. 3(b), in which the change of the integrated intensities is recorded with respect to the holding time. Thus the $\alpha-\beta$ reconstructive transition takes place with depending both on temperature and time. The time dependence at various temperatures of the Bragg reflections 312 in phase $I_{\alpha}$ of $\alpha$-sequence is summarized in Fig. 4. The part of the $\mathrm{Rb}_{2} \mathrm{MoO}_{4}$ crystals, which has transformed from the $\mathrm{I}_{\alpha}$ phase to the $\mathrm{III}_{\beta}$ phase once, maintained the structures of $\beta$-sequence.

However, these crystals suddenly returned to the previous $\beta-\mathrm{K}_{2} \mathrm{MoO}_{4}$ type structure (phase $\left.I_{\alpha}\right)$ at room temperature frequently [6]. The variation of the total mass before and after the phase transition was not observed. From the results of our study, the newly established transition sequence of $\mathrm{Rb}_{2} \mathrm{MoO}_{4}$ is summarized in Fig. 5 .

\section{Funding}

The present work was partly supported by the collaborative research project of Materials and Structures Laboratory, Tokyo Institute of Technology, and by a research grant from the Yamaguchi University Foundation. 


\section{References}

1. M. Iizumi, J. D. Axe, G. Shirane, K. Shimaoka, Structural phase transition in $\mathrm{K}_{2} \mathrm{SeO}_{4}$. Phys. Rev. B 15, 4392-4411 (1977).

2. I. Etxebarria, J. M. Perez-Mato, G. Madariaga, Lattice dynamics, structural stability, and phase transitions in incommensurate and commensurate $\mathrm{A}_{2} \mathrm{BX}_{4}$. Phys. Rev B 46, 2764-2774 (1992).

3. H. Shigematsu, Y. Akishige, H. Mashiyama, T.Tojo, H. Kawaji, T. Atake, T. Matsui, Heat Capacity, X-Ray scattering and neutron scattering studies in $\mathrm{Rb}_{2} \mathrm{SeO}_{4}$. J. Korean Phys. Soc. 46, $235-238$ (2005).

4. H. Shigematsu, Y. Akishige, T. Matsui, T. Tojo, H. Kawaji, T. Atake, Structures and Phase Transitions in $\left(\mathrm{K}_{1-x} \mathrm{Rb}_{x}\right)_{2} \mathrm{SeO}_{4}$. J. Thermal Analysis and Calorimetry 81, 555-558 (2005).

5. I. L. Yanchuk, A. Jorio, P. Saint-Gregoire, Thermodynamics of the incommensurate state in $\mathrm{Rb}_{2} \mathrm{WO}_{4}$ : The Lifshitz point in A2BX4 compounds. Phys. Rev B 61, 3147-3150 (2000).

6. H. Shigematsu, K. Nomura, K. Nishiyama, T. Tojo, H. Kawaji, T. Atake, Y. Kawamura, T. Miyoshi, Y. Matsushita, M. Tanaka, H. Mashiyama, Structures and phase transitions in $\mathrm{Rb}_{2} \mathrm{MoO}_{4}$ and $\mathrm{Rb}_{2} \mathrm{WO}_{4}$. Ferroelectrics 414, 195-200 (2011).

7. H. Shigematsu, K. Nishiyama, Y. Kawamura, H. Mashiyama, Neutron and X-ray Scattering Studies of $\mathrm{Rb}_{2} \mathrm{CoCl}_{4}$ and Successive Phase Transition in $\mathrm{A}_{2} \mathrm{BX}_{4}$-type Crystals. J. Phys. Soc. Jpn. 83, 124601 (2014).

8. H. Mashiyama, H. Shigematsu, Phase transitions and the quantum effect in $\mathrm{A}_{2} \mathrm{BX}_{4}$-type ferroelectric crystals. Ferroelectrics 511, 5-11 (2017). 


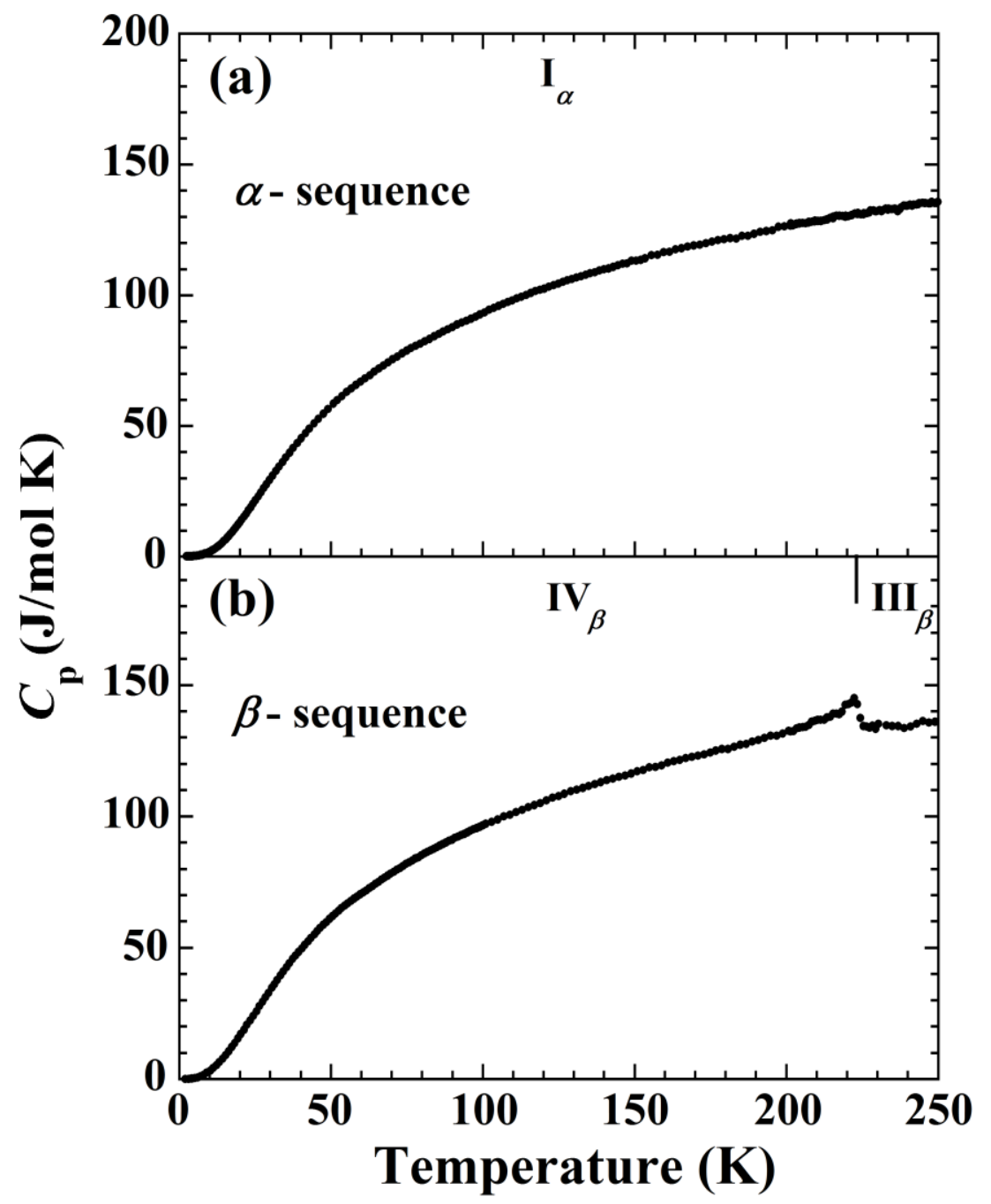

Figure 1. Molar heat capacity measured in as-grown $\mathrm{Rb}_{2} \mathrm{MoO}_{4}$ (a) and annealed $\mathrm{Rb}_{2} \mathrm{MoO}_{4}(\mathrm{~b})$. 


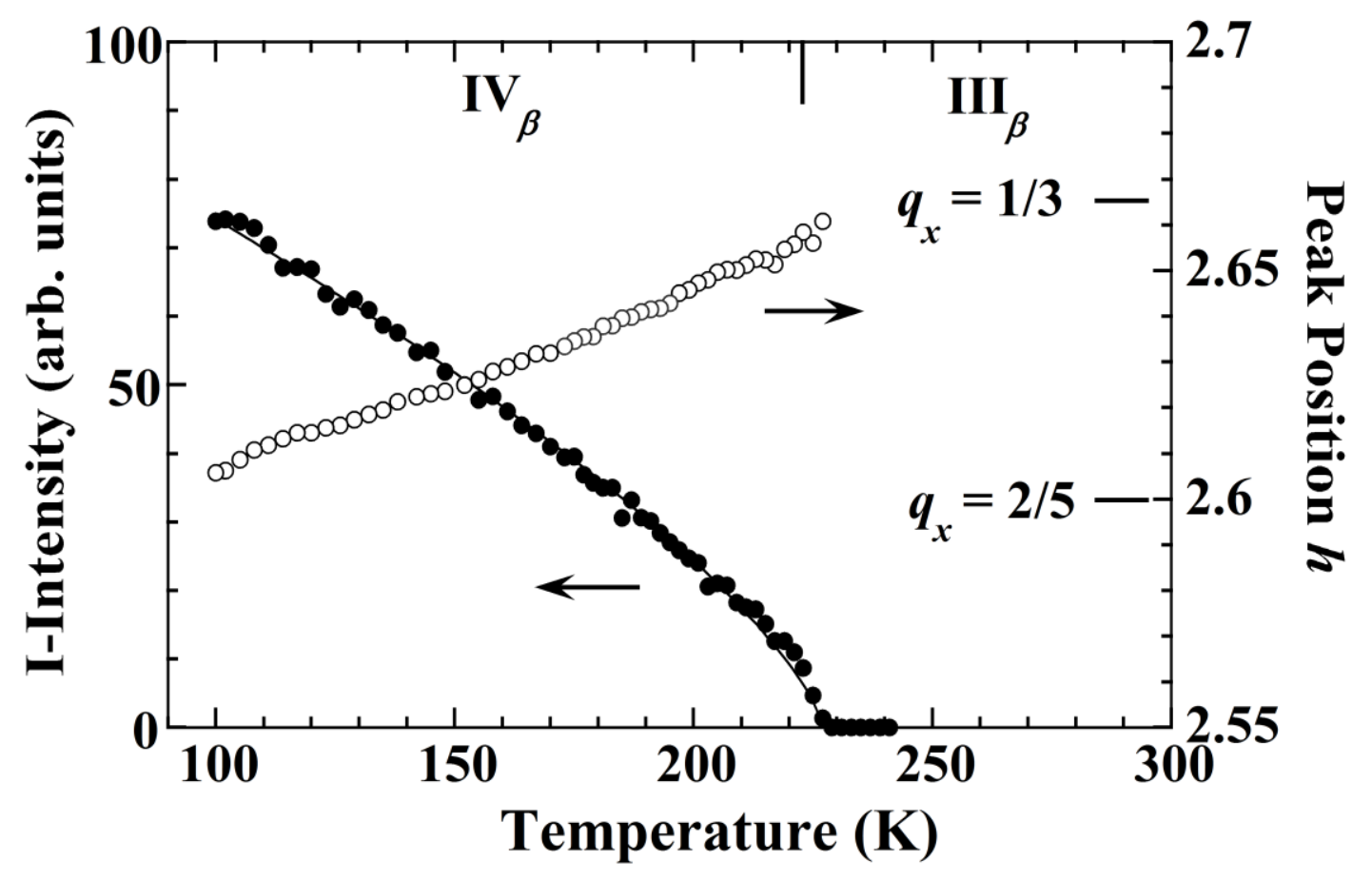

Figure 2. The temperature dependence of the integrated intensity and the satellite position of the $h 32$ superlattice reflection in phase $\mathrm{IV}_{\beta}$ for annealed $\mathrm{Rb}_{2} \mathrm{MoO}_{4}$. 

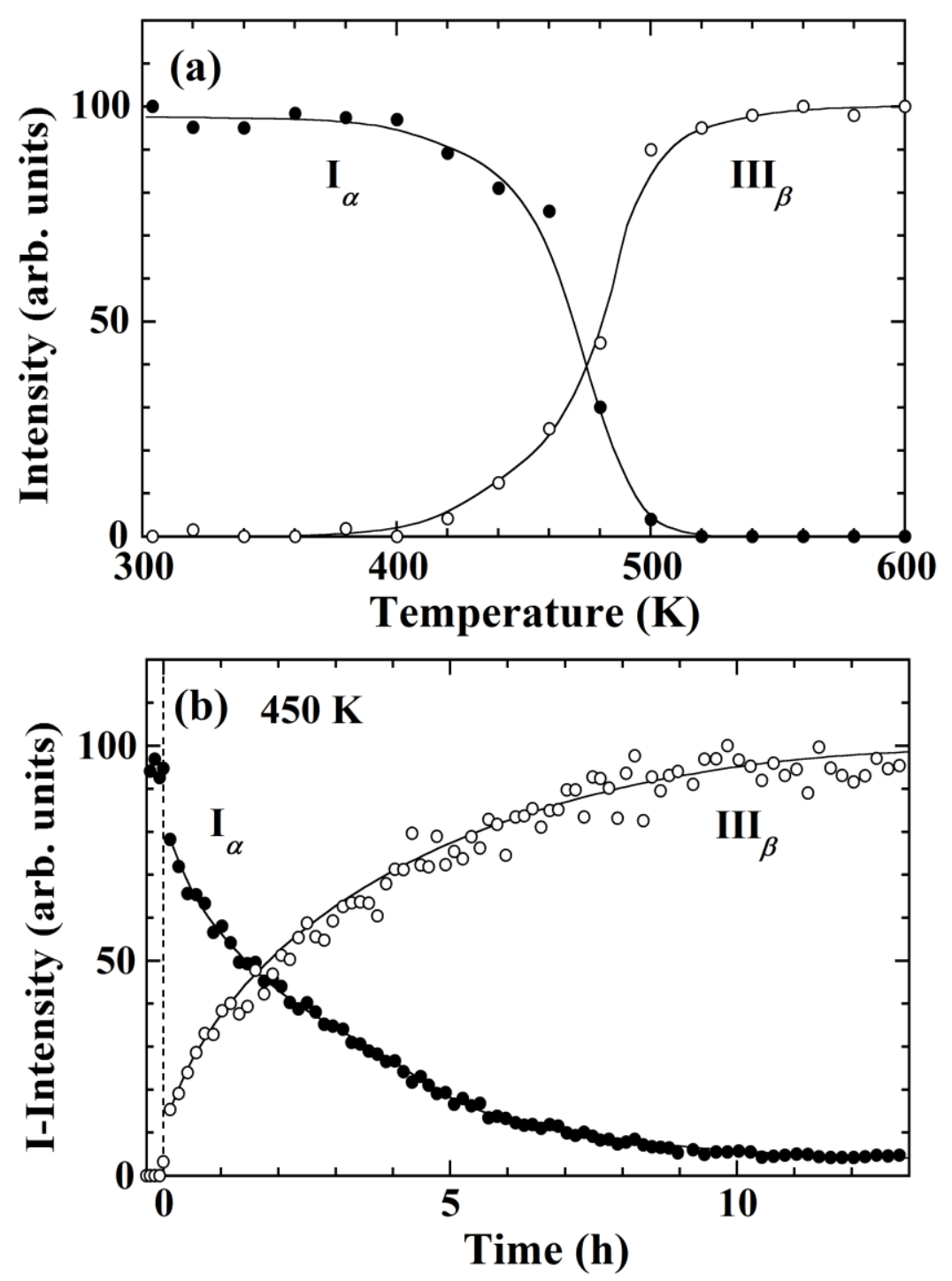

Figure 3. The gradual structure transformation of $\mathrm{Rb}_{2} \mathrm{MoO}_{4}$ from the phase $\mathrm{I}_{\alpha}$ of the $\alpha$ sequence to the phase $\mathrm{III}_{\beta}$ of the $\beta$-sequence. (a) On heating $10 \mathrm{~K} / \mathrm{min}$, the integrated intensity of the Bragg reflections 31-2 (phase $\mathrm{I}_{\alpha}$ of the $\alpha$-sequence) decreases, while 111 (phase $\mathrm{III}_{\beta}$ of the $\beta$-sequence) increases gradually. (b) On keeping temperature at $450 \mathrm{~K}$, the Bragg intensities of the phase $\mathrm{I}_{\alpha}$ and $\mathrm{III}_{\beta}$ counterchange, where the maximum intensity is scaled by 100 . 


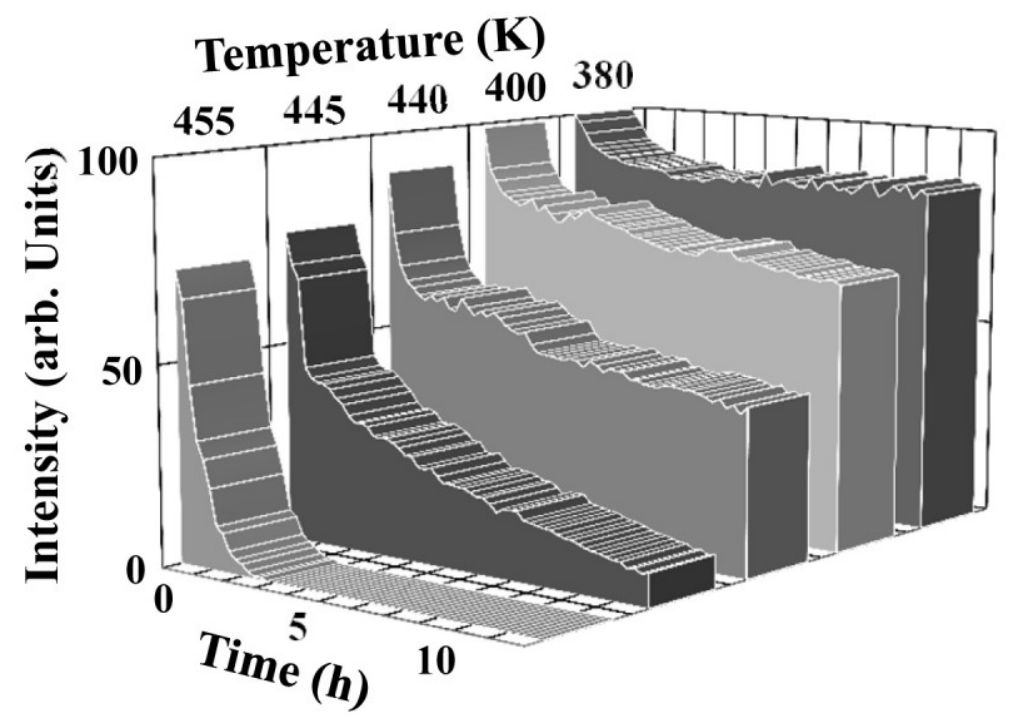

Figure 4. The time dependence of the integrated intensity of the Bragg reflection 312 in the phase $\mathrm{I}_{\alpha}$ of the a-sequence for the as-grown $\mathrm{Rb}_{2} \mathrm{MoO}_{4}$ at 380, 400, 440, 445, and $455 \mathrm{~K}$.

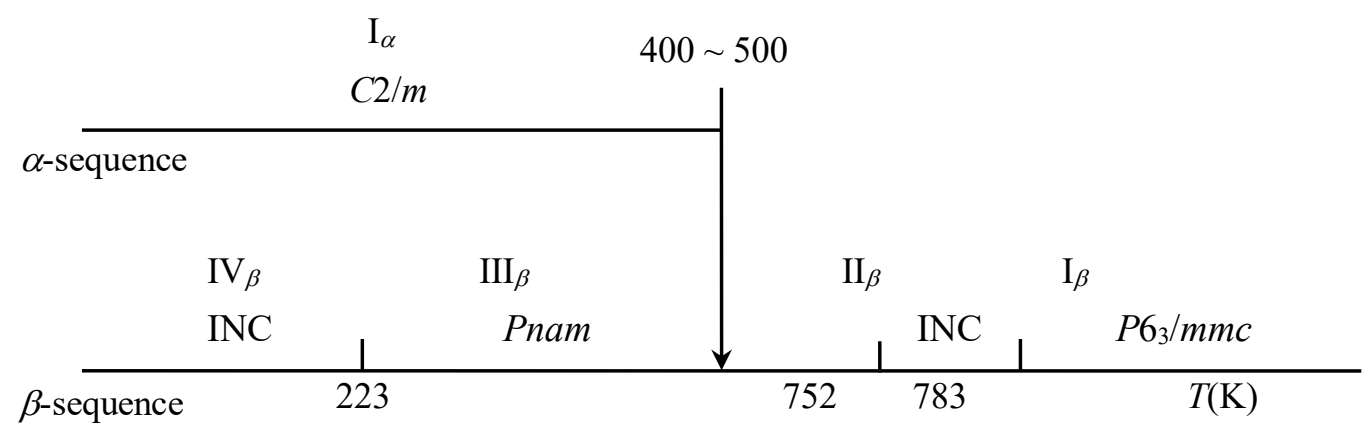

Figure 5. The revealed phase transition sequences of $\mathrm{Rb}_{2} \mathrm{MoO}_{4}$. 